\title{
Análisis de la siniestralidad en ciclistas 2008-2013
}

\author{
Dra. Ana Martí-Belda Bertolín \\ Investigadora INTRAS. Prof, Asoc. Universitat de València. España \\ Patricia Bosó Seguí, Ignacio Lijarcio Cárcel, Consuelo López Osma, \\ Investigador INTRAS. Universitat de València. España
}

\section{RESUMEN}

Los accidentes con víctimas ciclistas han sufrido un continuo ascenso en los últimos años. A fin de conocer cuáles son las características tanto del ciclista implicado en un accidente, como de las circunstancias en las que éste se ha producido, se ha realizado un estudio en el que se analiza la siniestralidad de los ciclistas registrada en España en el período comprendido entre 2008 a 2013. La investigación se ha realizado a partir de la base de datos de accidentes oficial de la Dirección General de Tráfico (base ARENA). En total se han analizado 25.439 accidentes con ciclistas implicados y 24.520 víctimas ciclistas. Los principales resultados de este estudio muestran que el número de accidentes de tráfico con ciclistas implicados, así como el número de ciclistas víctimas de accidentes, ha sufrido un ascenso continuado. Es en zona urbana donde se registran la mayoría de estos accidentes y víctimas, aunque los fallecimientos se producen en su mayoría en vías interurbanas (78,3\%). El factor concurrente más habitual en los accidentes de los ciclistas ha sido la distracción $(89,3 \%)$ mientras que el tipo de accidente más frecuente en el que se ven implicados es la colisión frontal/frontolateral (40,9\%). El perfil de la víctima ciclista es hombre (84’2\%), de 37,07 años y de nacionalidad española $(83,6 \%)$. Vistos estos resultados, un análisis exhaustivo de la accidentalidad del ciclista es clave para evaluar su evolución y los factores que influyen en ella, de modo que se consiga aumentar la seguridad para este tipo de transporte.

\section{INTRODUCCIÓN}

\subsection{La bicicleta como medio de transporte}

Las bicicletas se han convertido en los últimos años en un medio de transporte alternativo, utilizado cada vez por un número mayor de usuarios, no sólo como parte de su tiempo libre o como un deporte, sino también para los desplazamientos que realizan diariamente. Esto ha sido debido en parte a varios hechos, entre los que cabe destacar la creación de infraestructuras para circular, las prestaciones que ofrece la bicicleta, su facilidad de uso, su comodidad, el ser un trasporte saludable, lo económico de su uso o al gran número de ciudades españolas que cada vez con más frecuencia, ofertan un "préstamo de bicicletas".

Todo ello ha dado lugar a un incremento del uso de la bicicleta, que ha traído consigo, entre 
otras cosas, el aumento de incidentes y accidentes en las ciudades, donde todavía no existe una plena convivencia en la vía pública entre las bicicletas y los vehículos a motor. La bicicleta ya es el medio de transporte habitual para muchos españoles. Tanto es así, que en ciudades como Valencia, Vitoria o Zaragoza, en la actualidad más del 45\% de sus habitantes utilizan al menos una vez a la semana este medio de transporte.

A pesar del citado auge en el uso de las bicicletas en nuestro país, existen países europeos que cuentan con una tradición mucho más larga en lo que respecta a la utilización de este medio de transporte y son considerados como pioneros en el uso de la bicicleta y la cultura que rodea a este tipo de vehículo.

\subsection{Accidentalidad de las bicicletas}

A diferencia de los datos de accidentalidad generales, en los que se observaba un descenso progresivo hasta el año 2011, los accidentes de bicicletas con víctimas han sufrido un continuo ascenso desde 2008 hasta 2013 (gráfica 1), periodo en el que se centra este estudio.

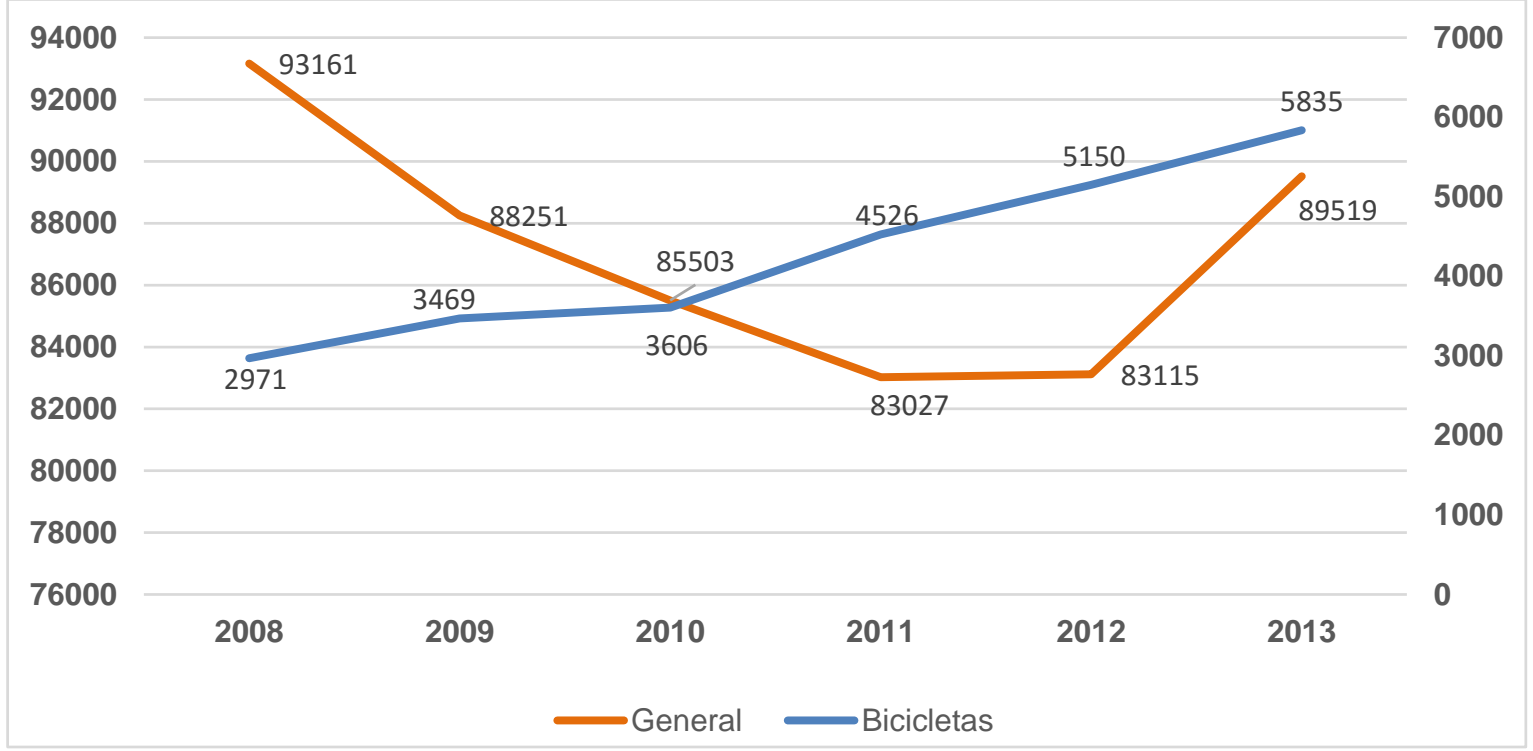

\section{Gráfica 1. Evolución de los accidentes con víctimas en España 2008-2013.}

En términos generales, los datos globales de los últimos años muestran cómo la siniestralidad vial se ha visto notablemente reducida, llegando a alcanzar mínimos históricos. Sin embargo, en el caso concreto de las bicicletas, el número de muertos que se ha registrado a lo largo de los últimos años ha sido variable.

En concreto, según los datos oficiales ofrecidos por DGT en el año 2013, los usuarios de bicicletas se vieron implicados en 5.835 accidentes en los que fallecieron 69 ciclistas y resultaron heridos graves 646 y fueron 4.779 los heridos leves. Atendiendo al lugar donde ocurrieron dichos accidentes, el 73\% se registró en vías urbanas, donde se produjeron el $72 \%$ del total de los heridos leves y el 54\% de los graves. No obstante, fue en las vías interurbanas donde se produjeron un mayor número de ciclistas fallecidos (45). 


\section{OBJETIVOS}

Objetivo General:

- Analizar la evolución y describir las características de todos los accidentes con víctimas donde se hallan implicadas bicicletas, en el período 2008-2013, dentro del territorio español, tanto en las vías urbanas como en las interurbanas.

Principales objetivos específicos:

- Recoger y analizar los datos completos de accidentalidad (todos los accidentes con víctimas que se hayan producido) en el colectivo de ciclistas durante el periodo que va del año 2008 al año 2013.

- Analizar las causas de accidentalidad de los siniestros de los ciclistas españoles relacionados con los factores humanos, el vehículo y el entorno.

- Identificar la tipología de los accidentes de los ciclistas registrados en las vías urbanas y en las interurbanas.

- Conocer los grados de lesividad de los ciclistas, los sistemas de seguridad y las principales zonas afectadas en el siniestro.

- Describir la situación espaciotemporal en la que se producen los accidentes viales de los ciclistas.

\section{METODOLOGÍA}

El presente estudio se ha realizado mediante la explotación de los datos de accidentes con víctimas, registrados en la Dirección General de Tráfico a través del "Cuestionario estadístico de accidentes de circulación con víctimas" y posteriormente recogidos en la base oficial de datos ARENA.

La explotación y análisis de los datos se ha realizado sobre todos los accidentes con víctimas ocurridos entre los años 2008 y 2013. En concreto, se han analizado 25.439 accidentes con ciclistas implicados y 24.520 víctimas ciclistas resultantes de dichos siniestros en los años mencionados. El análisis de los accidentes se ha llevado a cabo teniendo en cuenta todos aquellos siniestros en los que al menos uno de los implicados fue un ciclista, a pesar de que éste pudiera no haber resultado lesionado. Por otra parte, el análisis de las víctimas de accidentes comprende a los heridos leves, graves y fallecidos a 24 horas, pues no se dispone de los datos consolidados de muertos a 30 días del total del periodo objeto de estudio.

Se han realizado análisis estadísticos univariados para obtener una valoración global de la incidencia de cada factor o variable, en términos absolutos y relativos. Para ello, se han tenido en cuenta las siguientes variables:

- Variables temporales: mes, hora, día de la semana y tipo de día.

- Variables ambientales/entorno: provincia, zona, tipo de vía, anchura de calzada y carril, marcas viales, arcenes, elementos de seguridad, accidentes registrados fuera de 
intersección (rectas, curvas suaves, curvas señalizadas, etc.), superficie, luminosidad, factores atmosféricos, restricción de visibilidad, señalización de peligro y densidad de la circulación.

- Variables sobre factores concurrentes y tipo de accidente: tipología del accidente registrado, factores concurrentes y su relación con las principales variables de interés.

- Variables relativas al ciclista: análisis de las variables referentes a las víctimas ciclistas como: sexo, edad, nacionalidad, lesividad, motivo de desplazamiento, desplazamiento previsto, horas de conducción continuada, utilización de accesorios de seguridad, localización de las lesiones más graves, condiciones psicofísicas del ciclista, presuntas infracciones del ciclista, presuntas infracciones sobre velocidad y acción del ciclista en el momento del accidente.

Por otra parte, se realizaron una serie de análisis bivariados o multivariados mediante tablas de contingencia, donde se cruzaron las variables que se consideraron de especial interés teniendo en cuenta las significaciones estadísticas, en función del análisis de las puntuaciones obtenidas en los residuos ajustados estandarizados. Por tanto, en el informe se muestran sólo aquellos resultados que fueron estadísticamente significativos y cuya hipótesis de independencia entre las variables analizadas ha sido contrastada mediante el estadístico Chi cuadrado $\left(\mathrm{X}^{2}\right)$.

\section{RESULTADOS}

4.1. Evolución general de la accidentalidad ciclista: accidentes y víctimas implicadas. El número de accidentes de tráfico con ciclistas implicados ha sufrido un ascenso continuado a lo largo del periodo 2008-2013, acumulando un total de 25.439. Para el mismo periodo de tiempo y también ha aumentado el número de ciclistas víctimas de accidentes de tráfico hasta un total de 24.520 víctimas.

En cuanto a la gravedad de las víctimas resultantes, el aumento de las cifras se ha producido especialmente en el número de heridos graves y leves. En cambio, en el periodo total analizado se observa una reducción significativa en el número de ciclistas fallecidos.

\subsection{Análisis de las variables relativas a los accidentes con ciclistas implicados.}

\subsubsection{Análisis de la situación temporal del accidente con ciclistas implicados}

Los meses que registran mayores índices de accidentalidad son aquellos con mejores condiciones climatológicas. Julio es el mes en que mayor porcentaje de ciclistas resultan víctimas de un accidente y diciembre el mes con menor número de víctimas. Respecto a la lesividad, agosto es el mes más letal y el que más heridos graves registra.

Las horas más peligrosas para la ocurrencia de accidentes con ciclistas implicados son entre las 12 y las 15 horas $(28,4 \%)$, La franja horaria de entre las 7 y las 11 es la que acumula mayor número de fallecidos, aunque en términos relativos, la más peligrosa es la franja entre las 23 y las 11 horas. 
En cuando a la distribución semanal de los siniestros, en términos absolutos, tanto los accidentes con ciclistas implicados como el número de víctimas, se distribuyen de manera bastante homogénea. No obstante, el domingo es el día con menor accidentalidad y miércoles y jueves los días con mayor accidentalidad. En términos relativos, es el fin de semana el periodo más peligroso, pues sábado y domingo acumulan el mayor porcentaje de heridos graves y muertos.

Los festivos son los que registran mayor número de muertos y heridos graves. Los días laborales presentan un mayor número de accidentes en términos absolutos, aunque no registran un alto índice de muertos.

\subsubsection{Análisis de la situación espacial del accidente con ciclistas implicados}

Las provincias que mayor número de accidentes presenta son Barcelona (23\%), Madrid $(12,4 \%)$, Valencia $(8,3 \%)$ y Guipúzcoa $(4,1 \%)$, que a su vez son las provincias con mayor número de víctimas. En términos relativos, las provincias que mayor porcentaje de muertos han registrado son Cuenca (9,3\%), Zamora ( 8 '9\%) y Murcia $(7,1 \%)$.

En zona urbana se produce un mayor porcentaje de accidentes y víctimas. A su vez, la zona interurbana es la que registra un mayor porcentaje de muertos $(78,3 \%)$ aunque el porcentaje de heridos leves es superior en zona urbana $(71,1 \%)$.

El tipo de vía donde se produce más porcentaje de accidentes es -según la denominación oficial del parte de accidentes- "otro tipo de vía" (58,8\%), seguido de vías convencionales $(31,1 \%)$. Los porcentajes más elevados de víctimas en términos absolutos se dan en "otro tipo de vía" (56,5\%), vía convencional $(33,4 \%)$ y autopista $(3,7 \%)$. En términos relativos, las vías de servicio y autovías son las que registran un mayor porcentaje de muertos $(5,5 \%$ y 3,3\% respectivamente). En relación a las variables referentes a la vía, el análisis de los datos ha mostrado que las vías con más elementos de seguridad registran un mayor número de muertos, posiblemente debido a que éstas son vías que asumen mayores velocidades y por ellas circula mayor número de ciclistas.

Respecto a los accidentes y víctimas registrados fuera de una intersección, la mayoría se producen en rectas ( $84 \%$ y $82,4 \%$, respectivamente). No obstante, el mayor porcentaje de muertos se registra en curvas fuertes con señal y sin velocidad señalizada $(3,8 \%)$, seguida de curvas fuertes con señal y velocidad señalizada $(3,5 \%)$, y curvas suaves $(2,8 \%)$.

Se ha comprobado que se producen más accidentes y víctimas ciclistas cuando la superficie de la calzada está seca y limpia. En cuanto a la luminosidad de la vía, se registra un mayor número de accidentes y víctimas en pleno día, o por la noche habiendo iluminación suficiente. Sin embargo, en términos relativos, el número de muertos es significativamente mayor cuando la iluminación es insuficiente $(2,8 \%)$ o no hay iluminación $(10,3 \%)$. En 
cuanto a la restricción de visibilidad, se produce un mayor número de accidentes cuando no existe una restricción aparente de visibilidad $(75,4 \%)$, pero en el caso de existir, el mayor porcentaje de accidentes registra cuando el impedimento de visibilidad está producido por edificios $(14,1 \%)$. Lo mismo ocurre con el número de víctimas. En términos relativos, el mayor porcentaje de ciclistas muertos y heridos graves se produce por deslumbramiento $(6,7 \%)$ o cuando la visibilidad está disminuida por las características del terreno $(4,2 \%)$.

Respecto a los factores atmosféricos, la mayoría de los accidentes y víctimas suceden bajo condiciones de buen tiempo (92,4\% y 92,5\%, respectivamente). La condición atmosférica más peligrosa es la niebla intensa, con un porcentaje de $3,7 \%$ de muertos.

Se registra un mayor porcentaje de accidentes cuando la vía no está señalizada al ser innecesario $(44,9 \%)$. La tendencia es similar para el porcentaje de víctimas. Sin embargo, el número de muertos es mayor en vías que existe señalización $(1,8 \%)$, posiblemente por ser vías principales y por ello más usadas.

Se ha comprobado que hay un mayor porcentaje de accidentes y víctimas cuando la circulación es fluida (90\%). También bajo esta circunstancia se observa un porcentaje de muertos mayor $(1,8 \%)$, quizás debido a la mayor velocidad de los vehículos y el impacto sobre los ciclistas.

\subsubsection{Análisis del tipo de accidente y factores concurrentes}

El tipo de accidente más frecuente en el que se ven implicados los ciclistas es la colisión frontal/frontolateral (40,9\%), lo mismo que el número de víctimas en general $(42,2 \%)$. En cambio, el porcentaje de muertos es más alto en la colisión por alcance $(2,8 \%)$.

El factor concurrente más habitual en los accidentes de los ciclistas ha sido la distracción $(89,3 \%)$. Respecto al factor concurrente y el tipo de vía en la que se produce el accidente, el $35,7 \%$ de los siniestros causados por una distracción son en vías convencionales, también los accidentes por alcohol y drogas (41,4\%). A su vez, el 39,3\% de los siniestros por inexperiencia se registran en autopistas.

Sobre el tipo de accidente y la causa, los porcentajes, tanto de distracción, inexperiencia y alcohol-drogas son más altos en la colisión frontal/frontolateral. No obstante, existen elevados porcentajes de atropellos causados por la inexperiencia $(24,4 \%)$ y colisiones por alcance en los accidentes en los que hay implicación de alcohol y drogas (19,3\%).

Respecto a las variables temporales, sábados $(17,2 \%)$ y domingos $(17,6 \%)$ destacan por accidentes por alcohol y drogas. En cambio, durante la semana, los porcentajes de accidentes por inexperiencia y distracción son ligeramente más altos que los anteriores. En cuanto a la hora del día, hay un mayor porcentaje de accidentes entre las 19 y 22 horas por alcohol y drogas $(26,1 \%)$. Además son más frecuentes en mayo $(9,2 \%)$, junio $(9,2 \%)$, julio $(12,3 \%)$ y 
septiembre $(11,6 \%)$.

En lo referente al sexo de los ciclistas implicados, el factor concurrente con mayor presencia es la distracción, tanto en el caso de los hombres (90,3\%) como en las mujeres (85,1\%). En cuanto a la edad, en los siniestros con alcohol o drogas o inexperiencia como factores causantes, se observa un mayor porcentaje de ciclistas implicados entre 15 y 24 años (17,8\% y $21,2 \%$ respectivamente).

\subsection{Análisis de las variables relativas a los ciclistas víctimas de accidente.}

Los ciclistas víctimas de accidente son mayoritariamente hombres $(84,2 \%)$. Los hombres también presentan porcentajes de fallecidos más elevados que las mujeres (un 1,4\% frente a un $0,6 \%$ respectivamente). La nacionalidad de las víctimas es mayoritariamente española (83,6\%). La edad media de las víctimas es de 37,07 años. El mayor porcentaje de víctimas ciclistas se produce en el grupo con edades comprendidas entre los 25 y 34 años $(19,8 \%)$ pero las cifras de fallecidos son más elevadas en los rangos de edad de 75 a 84 años $(6,9 \%)$.

Con independencia de la edad del ciclista, la zona principalmente lesionada es siempre la cabeza (entre un $77,8 \%$ de los fallecidos de 0 a 14 años y un $56,7 \%$ en el caso de los fallecidos de 25 a 44 años) pero sólo el 51,2\% de los ciclistas llevaban puesto el casco en el momento del accidente. Los ciclistas con edades entre los 0 y 14 años y 15 a 24 presentan los porcentajes más bajos en utilización de este sistema de protección $(15,4 \%$ y 27,1\% respectivamente). Los ciclistas de Suiza, Gran Bretaña, Bélgica, Alemania y Holanda destacan por el alto porcentaje de utilización del casco cuando se produjo el accidente $(91,7 \%, 84,3 \%, 84 \%, 77,8 \%$ y $77,1 \%$ respectivamente). Hay un incremento de la utilización del casco a lo largo del periodo estudiado, que pasa de un $43,7 \%$ en el 2008 y a un $55 \%$ en el 2013. En carretera, el porcentaje de utilización de casco es mayor (76,8\%). En el caso de las zonas urbanas, más de la mitad de los ciclistas víctimas no lo utilizan $(67,2 \%)$.

De forma general, las partes más afectadas en los accidentes son las extremidades inferiores $(26,7 \%)$, las superiores $(22,2 \%)$ y la cabeza $(19,5 \%)$. Sin embargo el porcentaje es mayor en la cabeza $(27,5 \%)$ cuando las víctimas no utilizan el casco. Sobre las condiciones psicofísicas de las víctimas, el 98,5\% de los ciclistas muestra un estado aparentemente normal en el momento del accidente.

El 60\% de las víctimas ciclistas no han cometido ninguna infracción. Aquellas que sí han infringido lo hacen por aspectos como frenar, circular fuera de pista u otras (20\%) o por mantener una conducción distraída o desatenta $(9,3 \%)$.

Sobre la lesividad de las víctimas y la infracción cometida en el momento del accidente, el análisis de los datos muestran que los ciclistas que hacen una incorrecta utilización del alumbrado acumulan también un porcentaje más elevado de muertos $(8,1 \%)$, del mismo modo que aquellos que realizan un adelantamiento, circulan en sentido contrario o realizan 
giros incorrectos $(4,8 \%)$ y los que incumplen la norma marcada por diferentes señales, no mantienen el intervalo de seguridad o circulan de forma peligrosa $(2,9 \%)$.

El motivo del desplazamiento cuando se produjo el accidente es principalmente el ocio $(64,8 \%)$. A su vez, el desplazamiento más frecuente es el local de menos de 50 kilómetros $(89,1 \%)$. En el momento del accidente, el 47,2\% de las víctimas seguía la ruta. Se registra un mayor porcentaje de muertos cuando la víctima está girando a la derecha, izquierda o en $\mathrm{U}(3,6 \%)$.

\section{CONCLUSIONES}

Los datos obtenidos en el estudio permiten obtener una visión general de la problemática relacionada con el colectivo de los ciclistas. La movilidad de los ciclistas tiene que estar unida a la seguridad y para ello es necesario conocer en profundidad los datos relativos a ésta, objetivo de este estudio, y con ello contribuir a una progresiva evolución segura del uso de la de la bicicleta, como una alternativa de transporte seguro, sostenible y saludable, que repercute de forma directa en el bienestar no solo individual, sino del conjunto de una sociedad.

Para que se pueda seguir fomentando el uso de la bicicleta y se mejoren las condiciones de este tipo de desplazamiento, es importante tener en cuenta las características que hacen a países vecinos como Alemania, Países Bajos o Dinamarca pioneros en el fomento de la movilidad en bicicleta. En ellos, la educación vial y la formación son pieza clave para aumentar la seguridad ciclista. Diseñar, desarrollar e implementar programas de formación específicos es uno de los primeros pasos en el camino hacia un ciclismo seguro.

En relación con las infraestructuras, para aumentar el uso de la bicicleta, resulta imprescindible incrementar la protección del colectivo ciclista. Los datos obtenidos en el presente estudio muestran claramente como la zona urbana es la que acumula un mayor porcentaje de accidentes y víctimas. Aumentar la seguridad de los ciclistas pasa por la implantación de medidas como el aumento de calles con límites de velocidad $30 \mathrm{~km} / \mathrm{h}$, ciclovías, calles con sistemas de calmado de tráfico, incremento en las distancias de los carriles bici, carriles especiales para bicicletas que faciliten cruces y giros más rápidos, etc.

Por tanto, junto con la educación y formación, la inversión en infraestructuras, tanto en zona interurbana como urbana, orientadas a los desplazamientos de las bicicletas, lejos de interpretarse como una inversión aislada, debería considerarse en conjunto, valorando también el impacto que ésta podría tener en otros temas como la contaminación ambiental. 